ORIGINAL ARTICLE

\title{
Effect of incremental exercise on initiation and movement times in a choice response, whole body psychomotor task
}

\author{
T McMorris, S Delves, J Sproule, M Lauder, B Hale
}

Br J Sports Med 2005;39:537-541. doi: 10.1136/bjsm.2004.014456

See end of article for authors' affiliations

.....................

Correspondence to: Dr McMorris, University College Chichester, Centre for Sports Science and Medicine, College Lane, Chichester PO19 6PE, UK; t.mcmorris@ucc.ac.uk

Accepted 4 October 2004

\begin{abstract}
Objectives: To examine how exercise at moderate and maximal intensities affects performance on a choice response time, whole body psychomotor task.

Methods: Subjects ( $n=12$ ) were tested on a three-choice response time task, after rest and after exercise at $70 \%$ and $100 \%$ maximum power output $\left(\dot{W}_{\text {max }}\right)$. The dependent variables were time to begin forward momentum (initiation time) and time to complete the movement (movement time). Stride time for the first and second strides and number of strides to cover first $1.1 \mathrm{~m}$ were also measured. Blood lactate concentrations and heart rate were recorded before and after completion of each psychomotor test. The subjects subjectively assessed the amount of effort that they used to complete the task.

Results: Repeated measures analysis of variance showed a significant effect for initiation $\left(F_{2,22}=11.47\right.$, $\mathrm{p}<0.001)$ and movement times $\left(F_{2,22}=14.61, \mathrm{p}<0.001\right)$. Post hoc least significant difference $(L S D)$ tests showed that initiation time after exercise at $70 \% \dot{W}_{\max }$ was significantly faster than that in the other two conditions. Speed of initiation after rest was significantly quicker than that after exercise at $\dot{W}_{\max }$. For movement time, LSD tests showed that time after maximal exercise was significantly slower than that in the other two conditions. Stride time for the second stride showed a significant effect $\left(F_{2,22}=6.20, \mathrm{p}<0.01\right)$. LSD tests found that time after exercise at $\dot{W}_{\text {max }}$ was significantly slower in the other two conditions. Stepwise multiple regression analyses found that the increment of change, from rest, of lactate concentrations could significantly predict the increment of change in initiation $\left(R^{2}=0.40\right)$ and movement $\left(R^{2}=0.50\right)$ times.

Conclusions: Exercise affects a whole body task differently from purely cognitive tasks. Central factors are probably more important than peripheral factors.
\end{abstract}

l: many sports, the participant has to undertake exercise of various intensities and then perform a psychomotor skill. This skill often requires efficient control of the whole body. Often such skills must be carried out as quickly as possible. We are not aware of any research that has examined the effect of incremental exercise on the performance of such tasks. The primary purpose of this study was therefore to examine how exercise, at moderate and maximal intensities, affects the speed of performance of a gross psychomotor task requiring a fast response to a stimulus and quick movements through a slalom-type obstacle course

Several authors ${ }^{12}$ have claimed that exercise is a stressor and therefore will have the effect of increasing central nervous system (CNS) arousal levels. As a result, an inverted $\mathrm{U}$ effect of exercise on initiation and movement times during the performance of psychomotor skills has been hypothesised. Research, ${ }^{3-5}$ however, has failed to support this hypothesis. The types of psychomotor tasks used in all of these experiments required only a limited range of movement (11-44 cm) and only by the subject's arm.

When the response requires a whole body movement, however, one would expect different results. Therefore we hypothesised that maximal intensity exercise would have an inhibitory effect on movement time. Moderate intensity exercise should have a beneficial effect from warming up of the musculature and increasing the speed of nerve transmission within the peripheral nervous system.

The situation with regard to the initiation of the movement may be different from that for movement time. The stress of exercising maximally is thought to induce high levels of arousal in the CNS, resulting in a negative effect on performance. ${ }^{1}$ Surprisingly, research into purely cognitive tasks-for example, choice reaction time-has tended to show that heavy exercise induces faster responses than that after rest (see Tomporowski ${ }^{7}$ for a review). It may be that, when the response requires a whole body movement rather than just a finger press or a voice response, initiation time is affected differently. Therefore we hypothesised that initiation time after maximal intensity exercise would be significantly slower than that in the other two conditions.

With regard to the effect of moderate intensity exercise on initiation time, one would expect a positive result. Moderate intensity exercise is believed to elicit optimal levels of CNS arousal. ${ }^{12}$ This would aid reaction time. Therefore we hypothesised that initiation time after moderate intensity exercise would be significantly faster than that after rest. However, Kahneman ${ }^{8}$ has argued that, even at low levels of arousal, the individual can still focus on the task in hand because of what he calls cognitive effort. Cognitive effort is responsible for the allocation of CNS resources to the task. By focusing on the task, the individual can overcome low levels of arousal.

If cognitive effort is able to overcome the negative effects of exercise induced arousal, the increment of change in the individual's perception of the amount of cognitive effort that he/she produced, compared with his/her baseline perception (that before performing the psychomotor task after rest) $(\Delta \mathrm{CE})$, should be a significant predictor of similar increments of change in initiation time $(\Delta \mathrm{IT})$ and movement time $(\Delta \mathrm{MT})$. However, the physiological variables may prove to be more effective predictors of movement time than $\Delta \mathrm{CE}$.

Abbreviations: CNS, central nervous system; $\triangle C E$, change in cognitive effort; $\Delta \mathrm{HR}$, change in heart rate; $\Delta \mathrm{IT}$, change in initiation time; $\Delta \mathrm{LA}$, change in lactate concentration; $\Delta M T$, change in movement time; LSD least significant difference; $\dot{W}_{\text {max }}$, maximum power output 


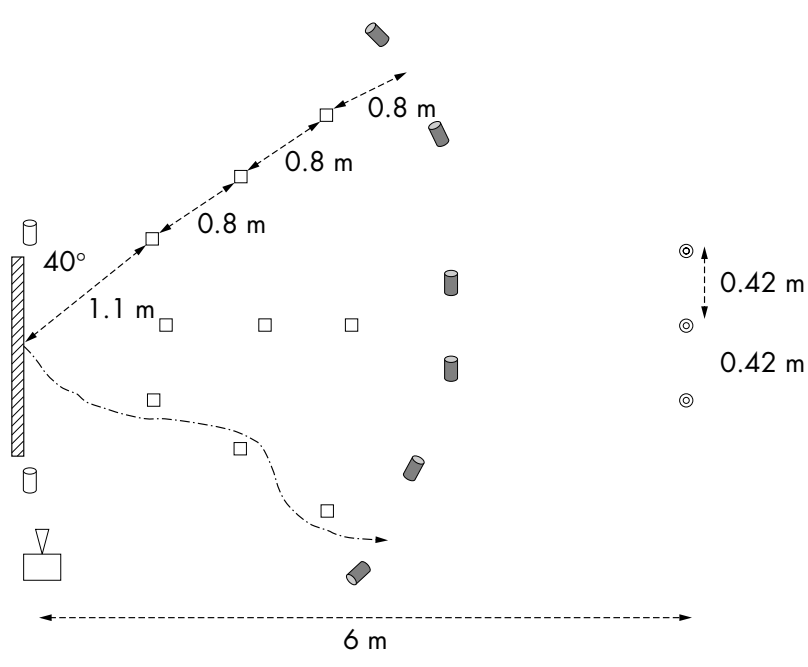

\begin{tabular}{|rl|}
\hline 9 & Starting line $1 \mathrm{~m} \times 0.05 \mathrm{~m}$ \\
0 & Light (forming part of light gate) $0.1 \mathrm{~m}$ high \\
$\odot$ & Light (forming part of light gate) $1.15 \mathrm{~m}$ high \\
$\square$ & Light stimulus, $1.9 \mathrm{~m}$ high \\
$\square$ & Cone with square base $0.15 \mathrm{~m}^{2}$ \\
$\ldots$ & Example of pathway taken by subjects \\
$\square$ & Peak Performance Technologies $200 \mathrm{~Hz}$ video camera
\end{tabular}

Figure 1 Diagrammatic representation of response task.

Therefore we examined the ability of the increment of change from rest in heart rate $(\Delta \mathrm{HR})$ and lactate concentration $(\Delta \mathrm{LA})$ to predict $\Delta \mathrm{IT}$ and $\Delta \mathrm{MT}$. $\Delta \mathrm{HR}$ is indicative of exercise intensity but is also affected by changes in arousal level. ${ }^{9}$ Similarly, as $\Delta$ LA is an indicator of fatigue, ${ }^{6}$ it may be able to predict $\Delta$ MT. $\triangle$ LA may also be a useful predictor of $\Delta \mathrm{IT}$. Previous research ${ }^{10}$ into reaction time has shown that performance after the lactate threshold has been reached is significantly faster than that before. Lactate concentrations follow an exponential curve during incremental exercise, and the point where there is a sudden increase is termed the lactate threshold. ${ }^{11}$

A secondary purpose of this study was to examine the effect of exercise on the nature of movement patterns. With increases in speed of nerve transmission after moderate intensity exercise, one would expect to observe quicker stride times. After maximal exercise, one might expect slower stride times, because of a loss of power. The individual may try to overcome slower stride times by taking several short strides.

To summarise, the primary purpose of this study was to compare the initiation and movement times of subjects in a whole body, choice response, time task after rest and after exercise at $70 \%$ and $100 \%$ maximum power output $\left(\dot{\mathrm{W}}_{\max }\right)$. The effect of the exercise on stride speed and frequency was also measured. The ability of $\Delta \mathrm{CE}, \Delta \mathrm{HR}$, and $\Delta \mathrm{LA}$ to predict $\Delta \mathrm{IT}$ and $\Delta \mathrm{MT}$ was calculated.

\section{SUBJECTS AND METHODS}

Subjects were paid male volunteers $(\mathrm{n}=12$; mean $(\mathrm{SD})$ age 24.24 (3.1) years, height $1.80 \quad(1.01) \mathrm{m}$, mass 84.56 $(12.53) \mathrm{kg})$. They signed an informed consent form and completed a medical questionnaire before taking part in the experiment. The institute ethics committee approved the study.

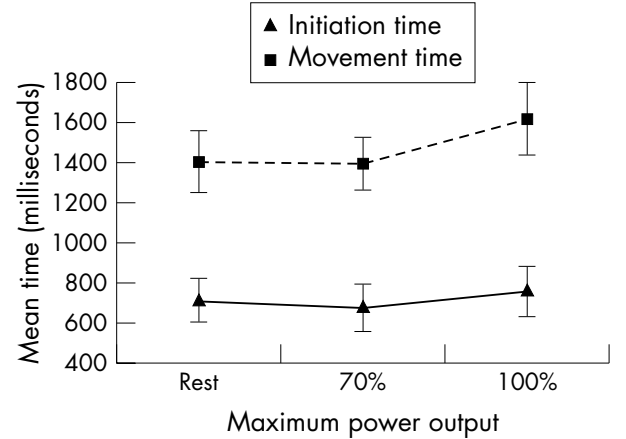

Figure 2 Mean initiation and movement times after rest and after exercise at $70 \%$ and $100 \%$ maximum power output.

\section{Psychomotor test}

Figure 1 shows the lay out of the test. Before undertaking the test, each subject was asked to stand with his toes touching the back of the start line, his head over the centre point of the line, and facing the display board. Subjects were instructed to run through the obstacles to their left when the L light was illuminated, those to their right when the $\mathrm{R}$ light was illuminated, and straight ahead when the C light was illuminated. Figure 1 shows the path to be taken when the $\mathrm{R}$ light was illuminated. The path when the L light was lit is a mirror of that for the $\mathrm{R}$ light. When the $\mathrm{C}$ light was illuminated the subject could begin by going right or left of the first central cone. From then on they had to follow a weaving pattern, as in the other two conditions. Subjects were given a verbal signal when a stimulus was to be presented. The period from the warning signal to presentation of the stimulus was randomised between 0.5 and 2 seconds. Presentation of the L, R, and C stimuli was also randomised, although each stimulus was presented three times in each set of trials that made up a test. This was necessary because the time taken to carry out the task may have been affected by whether the person had to move right, left, or centre. Subjects were not aware that this was the case. The psychomotor dependent variables were the time taken to initiate a forward movement (initiation time) and the time taken to complete the obstacle course (movement time). Initiation time was measured from the presentation of the stimulus to the breaking of the first light gate. Movement time was the time from the breaking of the first light gate to breaking the second gate. The biomechanical dependent variables were stride time for the first and second strides and number of strides to first cone. Each trial was recorded at $200 \mathrm{~Hz}$ using a high speed video camera (Peak HSC-200PM; Peak Performance Technologies, Centennial, Colorado, USA) and Panasonic G-5700 video recorder. Selected trials were played back through a Panasonic AG-MD830 videocassette recorder on to a Pentium II PC running Peak Motus Software 32 (version 2000) to determine the biomechanical variables.

\section{Procedure}

Before undertaking an habituation session on the psychomotor test, subjects sat for five minutes and their resting heart rate was recorded. They then undertook four sets of nine trials on the psychomotor test, with two minutes rest in between each set. After this they sat down until their heart rate reached resting level. They then carried out the $\dot{\mathrm{W}}_{\max }$ test.

In this test, subjects cycled on a Schoberer Rad Meßtechnik (SRM) cycle ergometer (Ingenieurbüro Schoberer, Fuchsend, Germany) at $70 \mathrm{rpm}$. The test started with a resistance of $100 \mathrm{~W}$ to which $6 \mathrm{~W}$ was added every 15 seconds (24 W/ min). Heart rate was continuously monitored by short range 


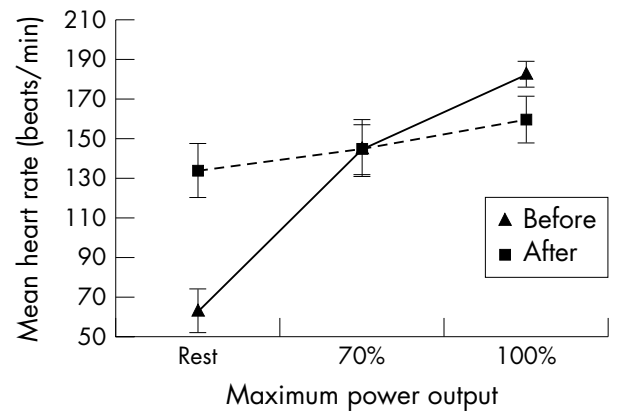

Figure 3 Heart rate before and after completion of the psychomotor test, after rest and after exercise at $70 \%$ and $100 \%$ maximum power output.

telemetry (Sports Tester PE-3000 monitor; Polar Electro, Kempele, Finland). The short range modem was linked to a computer which measured power output, pedal cadence, and heart rate every second. The test was terminated when the subject was unable to maintain the required pedal cadence. The $100 \%$ and $70 \% \dot{\mathrm{W}}_{\max }$ values were calculated; $70 \% \dot{\mathrm{W}}_{\max }$ is thought to be indicative of moderate intensity exercise. ${ }^{1}$

Forty eight hours after completing this test, the subject undertook the first of his psychomotor tests. On entering the laboratory, he was fitted with a heart rate monitor. In the rest condition, the subject sat on a chair for five minutes or until his heart rate reached baseline level, whichever was the longer. Then a blood sample was taken from the index finger of his right hand. Blood lactate concentrations were determined with an automated lactate analyser (2300 StatPlus analyzer; Yellow Springs Industries, Yellow Springs, Ohio, USA). After this, the subject carried out the psychomotor test. Immediately after the end of the ninth trial, his heart rate was recorded and a second blood sample for lactate determination was taken. The subject's perception of the cognitive effort used was ascertained using the effort subscale of the National Aeronautics and Space Administration task load index. ${ }^{12}$

In the $70 \% \dot{\mathrm{W}}_{\max }$ condition, the initial procedure was the same, but, when the subject reached resting heart rate, he began cycling. The cycling protocol was exactly the same as in the $\dot{W}_{\text {max }}$ test except that the subject only cycled until he reached $70 \% \dot{W}_{\max }$. In the last 30 seconds of the cycling, a blood sample for lactate determination was taken from the right index finger. As soon as he had finished cycling, the subject dismounted and took up the starting position. The set of nine trials began as close to 20 seconds after completion of the cycling as possible. Heart rate, lactate concentration, and perceived cognitive effort score were recorded immediately after the end of the test, as in the rest condition. The procedure for the $\dot{W}_{\text {max }}$ condition was identical, except that cycling continued until $\dot{W}_{\max }$ had been reached.

The order of presentation of the rest, $70 \%$, and $100 \% \dot{W}_{\max }$ conditions was counterbalanced. The order in which each of the movements-left, right, or centre-had to be made was divided into three sets. The order in which each of the sets was taken by the subjects with regard to exercise condition and order of presentation were also counterbalanced.

\section{Statistical analysis}

Initiation time and movement time in each of the three conditions were compared by a repeated measures analysis of variance. Speed of first and second strides to the first cone were also compared using repeated measures analysis of variance. Effect sizes were measured by the $\eta^{2}$ method. Fisher's least significant difference (LSD) was used as a post hoc measure. A series of $\chi^{2}$ goodness of fit tests were carried out to examine number of strides to first cone in each of the conditions. Cognitive effort after each of the psychomotor tests was compared by repeated measures analysis of variance. Heart rates and lactate concentrations before and after performance on the psychomotor test were compared using $2 \times 3$ (before/after $\times$ exercise condition) repeated measures analysis of variances. The ability of $\Delta \mathrm{CE}$ and $\Delta \mathrm{HR}$ before and after the psychomotor test and $\Delta \mathrm{LA}$ before and after the test to predict $\Delta \mathrm{IT}$ and $\Delta \mathrm{MT}$ were determined by stepwise multiple regression analysis. As subjects were included more than once in the regressions, the inflation factor of Scott and Holt ${ }^{13}$ was applied. This corrects for the non-independence of the sample.

\section{RESULTS}

Figure 2 shows mean initiation times for each condition. Repeated measures analysis of variance showed a significant effect for initiation time $\left(F_{2,22}=11.47, \mathrm{p}<0.001, \eta^{2}=\right.$ $0.51)$. Post hoc LSD showed that initiation of response after exercise at $70 \% \dot{W}_{\max }$ was significantly faster than that in the other two conditions. Speed of initiation after rest was significantly quicker than that after exercise at $\dot{W}_{\text {max }}$. A significant effect for movement time was also shown $\left(F_{2,22}=14.61, \mathrm{p}<0.001, \eta^{2}=0.57\right)$. LSD tests found that movement time after maximal exercise was significantly slower than that in the other two conditions, which did not differ significantly from one another. Figure 2 shows mean times.

Mean stride times for the first stride after rest, $70 \%$ and $100 \% \dot{W}_{\max }$ were 386 (87), 386 (94), and 403 (80) milliseconds respectively. Repeated measures analysis of variance showed no significant effect of exercise. Stride times for the second stride were 307 (84) milliseconds after rest, 309 (63) milliseconds after $70 \% \dot{W}_{\max }$ and 371 (111) milliseconds after $\dot{W}_{\text {max }}$. Repeated measures analysis of variance showed a significant effect $\left(F_{2,22}=6.20, \mathrm{p}<0.01, \eta^{2}=\right.$ $0.36)$. Post hoc LSD found that stride time after maximal intensity exercise was significantly slower than in the other two conditions, which did not differ significantly from one another. The $\chi^{2}$ tests showed that the number of strides to the first cone was not significantly affected by exercise.

Figures 3 and 4 show mean heart rates and lactate concentrations respectively before and after completion of each psychomotor test. A $2 \times 3$ (before/after $\times$ exercise condition) repeated measures analysis of variance showed significant main effects for heart rate before/after $\left(F_{1,11}=\right.$ 156.67, $\left.\mathrm{p}<0.001, \eta^{2}=0.93\right)$, and exercise condition $\left(F_{2,22}=\right.$ 438.79, $\left.\mathrm{p}<0.001, \eta^{2}=0.97\right)$. These were superseded by a significant interaction effect $\left(F_{2,22}=185.92, \mathrm{p}<0.001, \eta^{2}=\right.$ $0.94)$. Post hoc tests found that, in the rest condition, heart rate before was significantly lower than after. In the $70 \%$ $\dot{\mathrm{W}}_{\text {max }}$ condition there was no significant before/after effect. After exercise at $\dot{W}_{\text {max }}$, heart rate before the psychomotor

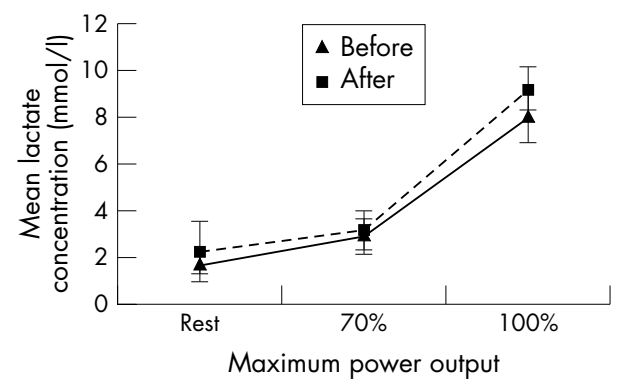

Figure 4 Lactate concentrations before and after completion of the psychomotor test, after rest and after exercise at 70\% and 100\% maximum power output. 
test was significantly higher than after. In the before conditions, there was a significant linear increase in heart rate from rest to $\dot{W}_{\max }$. In the after conditions, heart rate after $70 \% \dot{W}_{\max }$ was significantly faster than that after performance at $\dot{W}_{\max }$. For lactate concentrations, a $2 \times 3$ (before/after $\times$ exercise condition) repeated measures analysis of variance showed significant main effects for before/after $\left(F_{1,11}=17.72, \mathrm{p}<0.001, \eta^{2}=0.62\right)$ and exercise condition $\left(F_{2,22}=244.73, \mathrm{p}<0.001, \eta^{2}=0.96\right)$. Post hoc LSD tests found that there was a significant linear increase from rest to $\dot{W}_{\max }$. There was no significant interaction effect.

Mean perception of cognitive effort, scored on a 0-20 scale, after performance in the rest, $70 \%$ and $100 \% \dot{W}_{\max }$ conditions were 15.34 (4.54), 14.16 (5.00), and 17.66 (2.68) respectively. Repeated measures analysis of variance found no significant condition effects.

Initially it was intended to examine the ability of perception of $\triangle \mathrm{CE}, \Delta \mathrm{HR}$ before and after completion of the psychomotor test, and $\Delta \mathrm{LA}$ before and after to predict $\Delta \mathrm{IT}$ and $\Delta \mathrm{MT}$. However, $\Delta \mathrm{HR}$ before completion of the psychomotor test correlated highly with $\Delta$ LA before and after $(r=$ 0.73 and 0.71 respectively), and $\Delta$ LA before and after correlated significantly with one another $(r=0.88)$. Therefore, collinearity was a problem. To overcome this, only $\triangle \mathrm{HR}$ and $\triangle \mathrm{LA}$ after the tests and $\triangle \mathrm{CE}$ were used in the analysis. Stepwise multiple regression, with $\Delta \mathrm{IT}$ as the dependent variable, found that $\Delta \mathrm{LA}$ was a significant predictor: $R^{2}=0.40(\mathrm{p}<0.001)$. With movement time as the dependent variable, $\triangle \mathrm{LA}$ was again a significant predictor: $R^{2}=0.50(\mathrm{p}<0.001)$. Administration of the inflation factor did not affect these results.

\section{DISCUSSION}

The results of this study on movement time do not provide support for the inverted $U$ hypothesis. Movement time after maximal intensity exercise was significantly slower than that in the other two conditions. Commonsense would suggest that this is to be expected. A build up of lactate and break down of neurotransmitters in the peripheral nervous system would result in such an effect. However, previous research has shown an improvement in movement time during maximal exercise. ${ }^{5}$ In that study, however, the exercise was undertaken by the legs, whereas the psychomotor task was performed by the hands and arms.

The fact that movement time after rest and moderate intensity of exercise did not differ significantly is surprising. Exercise at $70 \% \dot{\mathrm{W}}_{\max }$ should ensure an increase in blood flow, speed of nerve transmission, and a general warming up of the muscles, which would aid movement. ${ }^{6}$ However, it appears that this was of no help in this task. This is further supported by the fact that stride times for both first and second strides were not affected by moderate exercise. It is difficult to account for these results. The intensity of exercise was such that one would have expected a positive warm up effect. However, it is possible that the time taken to reach $70 \% \dot{\mathrm{W}}_{\max }$ (mean (SD) 4.16 (1.19) minutes) was too short for the full positive effects of moderate exercise to be elicited. Time has been shown to be an important factor in warm up. ${ }^{14}$

The results on initiation time also fail to provide support for the inverted $U$ hypothesis. Initiation time after maximal exercise was significantly slower than that in either of the other conditions. This differs from research examining the effect of exercise on purely cognitive tasks, which has tended to show that heavy exercise induces improved response times. ${ }^{7}$ However, the tasks used in those protocols required only a finger movement or a voice response. The only research involving a whole body response found no effect of exercise on initiation time. ${ }^{15}$ The authors of that study, however, questioned their own results arguing that the design allowed the subjects to make some recovery from the exercise.

The present results are intuitively appealing. Maximal intensity exercise is thought to induce high CNS concentrations of noradrenaline (norepinephrine), which would induce overarousal. ${ }^{1}$ This will result in speed of perception of the stimuli being inhibited. ${ }^{16}$ That this result differs from the research reviewed by Tomporowski ${ }^{7}$ may be because the preprogramming necessary for a finger depression or voice response is less complex than that for a whole body response.

The fact that initiation time after moderate exercise was significantly faster than after rest is as hypothesised. Increases in CNS arousal during exercise would explain these results. The results fail to support Kahneman's ${ }^{8}$ argument that cognitive effort can overcome the negative effects of low arousal. Such a conclusion may be a little premature, however, as cognitive effort scores for the two conditions were not significantly different. That $\Delta C E$ was not able to predict $\Delta \mathrm{IT}$, however, suggests that it may play little part in the performance of this variable.

The results for stride time demonstrate something of an anomaly. The first stride was not affected by exercise of any intensity. This may be due to the fact that this stride did not require much in the way of working against inertia. The subject merely lifted the foot and moved it forward through the air. However, the second stride showed a negative effect of maximal exercise. This is probably because the second stride required the subject to push off the rear leg and generate force. This would be inhibited by maximal intensity exercise. ${ }^{14}$ However, these results cannot be examined in isolation from other stride variables. There were no significant effects of exercise on number of strides to the first cone. These findings are similar to those of studies examining the effect of sports specific exercise on the performance of sports skills; only a limited effect on biomechanical variables was also shown. ${ }^{17}{ }^{18}$ It should be noted that, in those studies, exercise was not maximal.

The results of the multiple regression analyses raise some important issues. As stated earlier, the failure of $\triangle \mathrm{CE}$ to predict $\Delta \mathrm{IT}$ and $\Delta \mathrm{MT}$ suggests that cognitive effort can not overcome the negative effects of high arousal levels. The failure of $\Delta \mathrm{HR}$ to predict $\Delta \mathrm{IT}$ and $\Delta \mathrm{MT}$ is surprising. Heart rate has been shown to be an indicator of arousal level ${ }^{9}{ }^{19}$ and is obviously a good indicator of exercise output. However, when the stressor causing increases in arousal is exercise, it may be that the heart rates elicited are poor predictors because the emotional and physical responses interact in such a way that heart rate becomes an unreliable predictor.

The ability of $\Delta \mathrm{LA}$ to predict $\Delta \mathrm{MT}$ is not really surprising. Changes in lactate concentrations are indicative of increases in exercise intensity, with its resultant depletion of neurotransmitters and increase in feelings of distress. ${ }^{6}$ That $\Delta \mathrm{LA}$ significantly predicted $\Delta \mathrm{IT}$ is of greater interest. Initiation time requires central (stimulus detection) and peripheral (time of first stride) inputs. One would have expected both of these factors to be affected by exercise, and $\Delta \mathrm{IT}$ to be the result of a combination of the central and peripheral variables. However, time for the first stride was not significantly affected by exercise. Therefore it would appear that the major factors causing changes in initiation time were central in nature.

That $\Delta \mathrm{LA}$, a peripheral biochemical measure, predicts such a change appears difficult to understand at first. However, other researchers have found the same. ${ }^{12}$ This may be because, during incremental exercise, plasma concentrations of lactate and catecholamines are highly correlated. ${ }^{1}$ It is thought that changes in plasma catecholamine concentrations influence similar changes in the CNS. ${ }^{1}$ Such changes should result in improvements in initiation time because 


\section{What is already known on this topic}

There appears to be no previous research on the effect of exercise on a response time task requiring a whole body response. Three studies have examined the effect of exercise on response time experiments requiring movement of the arm, but results are somewhat equivocal.

\section{What this study adds}

The results show that exercise affects a whole body task differently from purely cognitive tasks and that central factors are probably more important than peripheral ones. The results for initiation time are in line with allocatable resources theory predictions. The results for movement time showed no beneficial effect of warm up but a deterioration after maximal intensity exercise. Changes in lactate concentrations are good indicators of changes in initiation and movement times.

noradrenaline affects stimulus identification. This change in CNS concentrations may be due to peripheral changes stimulating the sympathetic nervous system resulting in secretion of catecholamines in the CNS. ${ }^{20}$

To summarise, as expected, maximal intensity exercise had a negative effect on initiation and movement times. This is different from previous research, which is probably because the movement required in this experiment was whole body rather than an arm movement as examined previously. The failure of moderate exercise to improve movement time suggests that warm up is of little use in the motor aspects of such a task. However, the improvement of initiation time from rest to moderate intensity exercise supports the idea that increases in exercise intensity induce rises in CNS arousal, resulting in faster initiation times. Thus warm up would have a positive effect on this aspect of performance. The failure of $\Delta \mathrm{CE}$ to predict either $\Delta \mathrm{IT}$ or $\Delta \mathrm{MT}$ questions the validity of the claims of Kahneman $^{8}$ that cognitive effort can overcome the negative effects of low arousal, at least when exercise is the stressor. That $\Delta \mathrm{LA}$ was a significant predictor of $\Delta \mathrm{MT}$ is not surprising. However, the ability of $\Delta \mathrm{LA}$ to predict $\Delta \mathrm{IT}$ is less straightforward and may indicate other biochemical changes that were not directly measured in this study.

\section{Authors' affiliations}

T McMorris, University College Chichester, Chichester, W Sussex, UK

S Delves, M Lauder, B Hale, University College Chichester

J Sproule, University of Abertay, Dundee, Scotland, UK

Competing interests: none declared

\section{REFERENCES}

1 Chmura J, Nazar K, Kaciuba-Uścilko H. Choice reaction time during graded exercise in relation to blood lactate and plasma catecholamines thresholds. Int J Sports Med 1994;15:172-6.

2 McMorris T, Graydon J. The effect of incremental exercise on cognitive performance. Int J Sport Psychol 2000;31:66-81.

3 Levitt S, Gutin B. Multiple choice reaction time and movement time during physical exertion. Res Q Exerc Sport 1971;42:405-10.

4 Williams LRT, Pottinger PR, Shapcott DG. Effects of exercise on choice reaction latency and movement speed. Percept Mot Skills 1985;60:67-71.

5 McMorris T, Tallon M, Williams C, et al. Incremental exercise, plasma concentrations of catecholamines, reaction time, and motor time during performance of a noncompatible choice response time task. Percept Mot Skills 2003;97:590-604

6 Åstrand PO, Rodahl K. Textbook of work physiology. New York: McGraw, 2002.

7 Tomporowski PD. Effects of acute bouts of exercise on cognition. Acta Psychol 2003; 1 12:297-394

8 Kahneman D. Attention and effort. Englewood Cliffs, NJ: Prentice-Hall, 1973.

9 Kennedy DO, Scholey AB. Glucose administration, heart rate and cognitive performance: effects of increasing mental effort. Psychopharmacology 2000;149:63-71

10 Chmura J, Krysztofiak H, Ziemba AW, et al. Psychomotor performance during prolonged exercise above and below the blood lactate threshold. Eur J Appl Physiol 1998;77:77-80.

11 Beaver WL, Wasserman K, Whipp BJ. Improved detection of lactate threshold during exercise using a log-log transformation. J Appl Physiol 1985;59:1936-40

12 Hart SG, Staveland LE. Development of NASA-TLX (Task Load Index): results of empirical and theoretical research. In: Hancock PA, Meshkati N, eds. Human mental workload. Amsterdam: North Holland Press, 1988.

13 Scott AJ, Holt D. The effect of two-stage sampling on ordinary least squares methods. J Am Stat Assoc 1982;77:848-54.

14 Chwalbińska-Moneta J, Kaciuba-Uścilko H, Krysztofiak H, et al. Relationship between EMG, blood lactate, and plasma catecholamine thresholds during graded exercise in men. J Physiol Pharmacol 1998;49:433-41.

15 McMorris T, Sproule J, Draper S, et al. Performance of a psychomotor skill following rest, exercise at the plasma epinephrine threshold and maximal intensity exercise. Percept Mot Skills 2000;91:553-62.

16 McMorris T, Myers S, MacGillivary WW, et al. Exercise, plasma catecholamine concentrations and decision-making performance of soccer players on a soccer-specific test. J Sports Sci 1999;17:667-76.

17 Davey $\mathbf{P}$, Thorpe RD, Williams C. Fatigue decreases skilled tennis performance. J Sports Sci 2002;20:311-18.

18 McGregor SJ, Nicholas CW, Lakomy HKA, et al. The influence of intermittent high-intensity shuttle running and fluid ingestion on the performance of a soccer skill. J Sports Sci 1999;17:895-903.

19 Hagemann D, Waldstein SR, Thayer JF. Central and autonomic nervous system integration in emotion. Brain Cogn 2003;52:79-87.

20 Genuth SM. The endocrine system. In: Berne MN, Levy BM, Koepen RM, et al, eds. Physiology. St Louis, MO: Mosby, 1998. 